\title{
Sociología y diseño: pistas, encuentros y des-encuentros"
}

\author{
Ponencia presentada en el X Congreso Nacional de Sociología
}

\author{
Mauricio Guerrero Caicedo** \\ (mauriciokempes@gmail.com)
}

\section{Introducción}

Esta ponencia está orientada a presentar los aspectos más relevantes de la exploración que ha venido realizando el Seminario de Sociología del Diseño (vinculado al Programa de Sociología y al Departamento de Humanidades de la Universidad Icesi) acerca de las relaciones que, en sociedad, se tejen entre la Sociología y el Diseño, cuando se asumen ambos como campos de prácticas que producen sentido acerca de lo social y sirven a su interpretación.

La inquietud que da origen a esta apuesta es, básicamente, la de abrir y explorar nuevos terrenos de estudio sociológicos. Simultáneamente, el énfasis en el diseño está fundamentado en la consciencia que tenemos de la urgente necesidad de adecuarnos a los cambios recientes del mundo social, uno de los cuales se manifiesta, precisamente, en la agudización de la preponderancia del diseño como práctica que moldea los hábitos de los agentes humanos e influye en sus decisiones (Latour 2008).

En este contexto, asistimos actualmente a lo que puede denominarse como una redimensión de lo social en el campo del diseño. La descripción y el abordaje crítico de dicho

\footnotetext{
* Ponencia presentada en el X Congreso Nacional de Sociología: Herencia y ruptura en la sociología colombiana contemporánea, realizado en noviembre 2, 3 y 4 de 2011 en Cali, Colombia.

** Investigador y profesor en la Universidad Icesi. Correo electrónico: mauriciokempes@gmail.com
} 
fenómeno desde la sociología se hacen, pues, necesarios, generan la oportunidad para explorarnuevos acercamientosmetodológicos y teóricos al estudio de lo social que, eventualmente, podrían enriquecer el espectro de la práctica sociológica.

En la confluencia de los acercamientos que a su objeto de estudio realiza el Seminario de Sociología del Diseño, éste adquiere el estatus de un campo de mediación social, en el que participan diversos agentes - entre los que se incluyen los objetos mismos de diseño, los usuarios, los diseñadores- cada uno de los cuales establece vínculos o relaciones dinámicos con los restantes. De estas matrices relacionales se desprenden los sentidos y significados que caracterizan a diferentes espacios de socialización que gozan de un papel ya tradicional en el desenvolvimiento de los procesos de habitación del dispositivo urbano.

Hasta el momento, nuestro objeto de investigación se ha centrado en un tipo específico de estos espacios de socialización: los Centros Comerciales o, recurriendo al término (singular, pero englobante) que se impone en el mundo académico y medra cada vez más en la práctica discursiva cotidiana, el Mall.

En estos espacios se ha puesto a prueba, por medio de un conjunto de estudios articulados entre sí, la pertinencia de nuestras intuiciones y desarrollos teóricos. Los resultados de este esfuerzo, hasta el momento, son alentadores, sobre todo en lo que respecta a la pertinencia de la introducción de la observación iconológica en el arsenal analítico del sociólogo.

Es a partir de la observación iconológica que se han producido, a lo largo de nuestro proceso como seminario, un conjunto de reflexiones y ejercicios de investigación que nos han permitido acceder a fuentes de información valiosas, entre las que ocupan un lugar primordial los objetos de diseño (Moles, 1975). Estas fuentes, sometidas al rigor iconológico, se transforman en elementos fundamentales para la decodificación de las diversas actualizaciones que se presentan en lo social a propósito de las dinámicas de relación entre el diseño y sus usuarios.

El abordaje iconológico de los objetos, en el marco de la descripción sociológica, nos dota de los datos necesarios para el establecimiento de patrones de relación estructurados sobre la base no sólo de lo inmediatamente observable en las interacciones entre los diversos agentes vinculados en una situación y en el marco de un espacio de socialización, sino, además, de la inclusión de valiosas pistas acerca de la forma en que la historia de dichos 
objetos, su dimensión material, las funciones para las que son estratégicamente destinados, y aquellas para las que son apropiados. De esta manera se sientan las bases para una rica caracterización de la forma en que la práctica del diseño y los objetos que ésta produce son modelizadas a través de sus diferentes usos sociales.

\section{Presupuestos teóricos y metodológicos generales del trabajo del Seminario de Sociología del Diseño de la Universidad Icesi}

Las relaciones entre sociología y diseño se han caracterizado, durante mucho tiempo por actualizarse de manera preferente en el seno de los estudios de consumo. A partir de esta perspectiva se han realizado valiosos aportes al estudio de las funciones sociales del diseño. Sin embargo, dada la naturaleza de dicho tipo de estudios, estos tienden a conferir al diseño el valor secundario de variable comprometida en las dinámicas del consumo.

Como resultado de lo anterior, el estudio propiamente sociológico del diseño como práctica social queda diferido o se le confía a los esfuerzos parciales de la comunicación social como disciplina (que indaga, a través del análisis de las formas de los objetos, por claves y constantes referidas a la manera en que una cultura determinada concreta su sentido del orden y sus ideas acerca de lo bello y lo útil); a los estudios de diseño (en buena medida orientados hacia la indagación y descripción formal de las dinámicas y constantes formales que en un momento y lugar determinados responden a la demanda social por objetos de diseño) (Sánchez, 2001); y a diversas tentativas que desde la semiótica y las disciplinas afines se establecen con miras a desentrañar los secretos de la manera particular en que los procesos de semiosis (Verón,1993) tienen lugar en, o son mediados por, esos mismos objetos de diseño.

El Seminario de Sociología del Diseño de la Universidad Icesi surge como resultado de la reflexión continuada de un grupo de profesionales ligados a las ciencias sociales y al campo del diseño a propósito de la situación arriba descrita. La búsqueda de opciones metodológicas y teóricas que permitieran articular las dimensiones del diseño como objeto de estudio que se encuentran comprometidas en los diversos tipos de investigaciones en los que éste adquiere el estatus de objeto primordial de la investigación, o de variable y dimensión constitutiva de otros objetos primarios, ha fungido, desde los 
inicios del seminario, como marco de referencia del desarrollo de un proceso académico que al presente ha producido un grupo de investigaciones pioneras en el contexto regional y nacional, articuladas principalmente en torno a los referentes fundamentales de "objetos de diseño" y al constructo heurístico que supone la asunción del fenómeno contemporáneo del Centro Comercial (Mall) como un macro-objeto de diseño en el que confluyen, a múltiples niveles (desde el dominio de los micro-objetos hasta el de su continente arquitectónico) diversas manifestaciones de esta disciplina, estableciendo entre sí un sistema de relaciones dinámicas e interactivas, en el seno del cual se actualizan constantementelas prácticassociales concretas que son posibilitadas, determinadas, mediadas e influenciadas por esos mismos objetos.

Este esfuerzo articulador se ha basado en el desarrollo de un abordaje del objeto de estudio caracterizado por un método sociológico de indagación modelizado principalmente en clave iconológica, pero también permeado de análisis semiológico e iconográfico, a través del cual lo que se reconstruye en el discurso académico es la forma en que los objetos de diseño sirven a la semiosis social y a las modelizaciones de la experiencia y las relaciones entre agentes humanos e inanimados que emergen en el momento del uso o la apropiación (De Certeau, 2000) de éstos por parte de sus usuarios.

Esta propuesta parte de la consideración de la función general del diseño -en tanto que práctica-, y los objetos en que ésta práctica se concreta, como mediadores sociales. En este sentido, las prácticas propias de la esfera del diseño se ubican, desde la perspectiva sociológica, en el seno mismo de la mayor parte de los intercambios que constituyen la configuración de las relaciones sociales en que los objetos cumplen el papel de agentes; y en las que simultáneamente, cumplen la función de significantes a partir de los cuáles se verifican las operaciones de semiosis en que estriba la significación que caracteriza a cada intercambio o relación particular. De esta manera, los objetos de diseño se asumen como dispositivos centrales en la emergencia de las dinámicas relacionales que dan forma a la materia de lo social, y subyacen a las distintas actualizaciones cotidianas de la operación fundamental (tanto para los sujetos-agentes operadores del diseño, como para el sistema social como un todo) de interpretación de la realidad.

Complementariamente, en el Seminario de Sociología del Diseño de la Universidad Icesi, los objetos de diseño son considerados, desde los presupuestos teóricos y metodo- 
lógicos que atraviesan los diferentes trabajos que se han realizado en el transcurso del mismo, como fuentes de información.

Este estatuto acordado a las diversas manifestaciones del diseño las inserta en el conjunto de las problemáticas relacionadas con las dinámicas de poder, entendido como subsistema relacional por medio del cual unas formas de interpretar el mundo; unos patrones de conducta (o de uso); y un conjunto de operaciones estratégicas -emanadas del campo de los expertos y especialistas bajo la especie de una gramática y de usos tácticos -característicos de los usuarios ajenos al campo de producción de sentido de dichos especialistas (De Certeau, 2000), actualizan la tensión cotidiana de las apropiaciones. Este proceso se manifiesta por medio de las enunciaciones espontáneas (haceres con lo ajeno, con lo dado) y, al mismo tiempo predeterminadas, a través de las cuales el diseño cumple su papel de mediador social.

\section{Sociología y Diseño asumidos como prácticas}

El equipo que conforma el Seminario de Sociología del Diseño estuvo consciente, desde el principio de sus actividades, de la necesidad de abordar su objeto desde la dimensión conceptual de las prácticas, entendidas como haceres y representaciones ligadas a las situaciones, los sistemas de relaciones y los intercambios entre los distintos agentes partícipes de una misma instancia de actualización de la materia de lo social.

Además de ello, se identificó la necesidad de dislocar el conjunto de representaciones tradicionales del investigador social que se traducen en lo que podría ser denominado el "imperialismo epistemológico" de la práctica sociológica, que consiste a menudo en olvidar las implicaciones que trae consigo el hecho de que la sociología misma sea una práctica.

Esa condición de la actividad sociológica, asumida de manera consciente, puede representar un valioso enriquecimiento en términos heurísticos para los investigadores. En este caso, desde esa perspectiva, la introspección del sociólogo y una buena dosis de distanciamiento con respecto a la forma en que su propia disciplina media y produce significados que reifican sentidos en el discurso académico, le permite acercarse al diseño con una mayor sensibilidad hacia las dinámicas complejas del tipo de mediaciones que éste lleva a cabo en el seno del sistema social. 
De esta manera se abre un horizonte de análisis en el que es posible no sólo abordar al diseño como un campo de producción de sentido, sino también, y al igual que la sociología que lo estudia, como un campo de interpretación de sentido. La elicitación de estas dos dimensiones de la semiosis propia del diseño en lo social, implica, además, la pregunta por la incidencia del diseño en lo social.

Con base en lo anterior, se ha establecido que el conjunto de las actividades académicas y de investigación que tienen lugar en el Seminario de Sociología del Diseño, tienen como rasgo en común el estar orientadas a aportar datos, elementos de análisis e hipótesis pertinentes, para el avance de la exploración intelectual en el terreno hasta ahora poco transitado que se sintetiza en la pregunta por las modalidades que reviste la inserción de lo social en el diseño, así como también por el carácter de la relación que se establece entre ambos términos de la ecuación "sociedad y diseño".

A esta exploración corresponde, metodológicamente, la que resulta de la inversión de los términos de la primera designación de la relación que nos interesa comprender en términos teóricos. El resultado de dicha inversión es la pregunta por las diversas modalidades en que el diseño mismo comporta una práctica social siempre que aparece como mediador entre los agentes conscientes de las relaciones sociales.

Este aspecto de la investigación es fundamental para la problematización de cualquier conjunto de prácticas discursivas que pretenda entronizar prácticas de diseño restringidas y específicas como eminentemente "sociales", a despecho de otras, a las que por contrapartida se les adjudicaría la función de servir a fines ajenos a aquellos que competen a las mediaciones entre los agentes que convergen en lo social, entre los que se cuentan los mismos objetos de diseño.

Una comprensión global de las diversas formas en que el diseño media la actualización de lo social, puede representar un campo fértil para futuras indagaciones sociológicas -y de innegable interés antropológico acerca de las dinámicas articuladas en la producción del conjunto de rituales y procesos de cambio que confluyen en la realización de la materia de lo social bajo la forma de prácticas concretas de consumo, selección y modalidades de apropiación de productos, espacios y situaciones (Delgado,1999). 


\section{La descripción sociológica}

La empresa de descripción sociológica del diseño en tanto que práctica que produce objetos que actúan como mediadores sociales comporta dos dimensiones de análisis complementarias. La primera de éstas es la que compete al estudio de los aspectos iconográficos propios de cada conjunto de objetos de diseño y de cada objeto en particular. Por esta vía se enriquece la visión sociológica del estudio al incorporar el análisis de las formas en que lo material y sus configuraciones cuentan como variables relacionales que agencian, promueven o inhiben las actualizaciones de las intenciones de los agentes humanos en el marco de sus interacciones sociales.

La segunda aproximación a las problemáticas que, desde la perspectiva sociológica son relevantes para el esfuerzo de elucidar las complejas funciones del diseño es la que indaga por las funciones que la dimensión iconológica de los objetos de diseño cumple en el entramado de los intercambios e interacciones entre los agentes humanos, y entre éstos y su entorno, el cual ha sido codificado e intervenido por operaciones de diseño en un nivel macro de la práctica, y es en sí mismo un producto del diseño.

Este tipo de abordaje del objeto de estudio hace posible situar a los objetos de diseño en el marco de las prácticas rituales que ellos mismos encarnan, así como también de aquéllas en las que actúan como mediadores, modelizadores, índices, íconos y símbolos $(\mathrm{Eco}, 2000)$ que son gestionados por los agentes humanos en sus prácticas cotidianas. De la elucidación de estas funciones depende, en gran medida, la comprensión de las dinámicas que subyacen a la forma en que se manifiestan al observador los órdenes que revisten dichas prácticas cotidianas, y la de la forma en que, a través de ellas, se reproducen, difunden, impugnan o modifican los valores que se encuentran implicados en éstas.

En síntesis, por esta vía, la mirada sociológica apunta al desvelamiento de las modalidades en que dos formas de poder, a saber: la que corresponde al poder de lo material y la que corresponde al poder de lo ritual se articulan y confluyen en la práctica del diseño, y operan a través de los objetos de diseño para influir en lo social y dotar de diversas modalidades de coherencia a las prácticas de otras esferas de producción de sentido (Heller, 1994). 
El Mall como macro objeto de diseño y los objetos de diseño al interior del Mall Los presupuestos de la investigación hasta aquí expuestos han servido de base teórica y metodológica para el desarrollo de un conjunto de proyectos centrados en el Macro-objeto Mall (o Centro Comercial). Esta elección ha sido motivada, en gran medida, por la creciente importancia que tienen estos dispositivos arquitectónico-comerciales en la determinación de la materialidad de la ciudad y de las prácticas de sociabilidad y consumo de los ciudadanos.

Tres investigaciones, interrelacionados y solidarios entre sí, se han realizado hasta el momento en torno este objeto y a la forma en que, en su interior, los restantes objetos de diseño en la escala micro se adecuan a unas lógicas y dinámicas de sistema en las que se pueden identificar un conjunto de constantes en lo que respecta a modalidades de uso.

En el primero de estos tres estudios se caracterizan las "Funciones, Usos y Significados de los Objetos de diseño en tres Centros Comerciales de la Ciudad de Cali". Estos tres centros comerciales son Unicentro, Cosmocentro y el Supercentro La de Calima.

El segundo estudio, titulado "Significados que los usuarios dan a los objetos de diseño en tres centros comerciales de Cali desde la perspectiva del consumo Cultural" introduce en la investigación la categoría analítica del significado (significación) conferido por los usuarios a los objetos de diseño en el marco de las relaciones cotidianas y los usos del espacio comercial en los mismos tres centros comerciales.

Por su parte, el tercer estudio, que responde a la denominación de "Usos y significados de la plaza en el espacio público y en el centro comercial. Análisis de un desplazamiento en una perspectiva social y política", se centra en la descripción, caracterización y comparación entre las modalidades de uso y apropiación-semiosis del espacio y los objetos de diseño que tienen lugar en dos versiones -una pública y otra privada- del mismo dispositivo arquitectónico urbanístico: la plaza.

A continuación, se describirán, en términos generales, los objetivos y los aspectos metodológicos propios de cada una de estas investigaciones.

Funciones, usos y significados de los objetos de diseño en tres centros comerciales de la ciudad de Cali

Este proyecto presenta una orientación descriptiva y comparativa, basada en la elección, como lugares de observación, de tres centros comerciales ubicados en diferentes 
zonas de estratificación socioeconómica en la ciudad de Cali. Aunque la metodología utilizada es principalmente cualitativa y de corte etnográfico, y se recurrió a la realización de entrevistas en profundidad, también se hizo uso de un instrumento cuantitativo diseñado para explicitar las tendencias generales que describen las relaciones entre usuarios-consumidores y objetos de diseño.

El objetivo del proyecto era permitir un acercamiento sociológico al estudio del diseño en sociedad a través de la indagación, en primera instancia, de las funciones a las que, en cada uno de estos espacios, se destinan -y las que cumplen- los objetos de diseño. Complementariamente se indagaron lo usos que se dan a estos objetos de diseño, y los significados que adquieren en el sistema del Mall.

Para ello se partió de una categorización de los objetos de diseño basada en tres modalidades de la práctica diseñadora: la arquitectónica, la propia del diseño industrial, y la que compete al diseño gráfico y publicitario. Dicha clasificación posteriormente se transformaría en un conjunto de tipologías de objetos de diseño, elaborada con arreglo a las características físicas de los mismos, que sirve de base al estudio de las prácticas sociales que cada tipo de objetos determina.

Tras la realización de esta operación de categorización de los objetos, en la que, siguiendo el modelo por Moles se los clasificó a partir del criterio de su relación con el cuerpo humano, y según su pertenencia a cada una de las dimensiones de la práctica del diseño arriba señaladas, se encontró que, en general, las funciones de los objetos, tanto a nivel micro como macro-objetual, se encuentran determinadas por: el pensamiento estratégico que da origen al centro comercial, el tipo de inserción socioeconómica que éste tiene en la cuadrícula de la ciudad, y por la forma propiamente material que les es propia.

Las combinatorias de estos elementos son diversas y el resultado de ellas es un estatuto diferente, según el centro comercial del que se trate, de los propios objetos de diseño. En este sentido, la tendencia general apunta a un hecho sociológico: el diseño tiende a cobrar mayor autonomía y relevancia como variable en la determinación de las prácticas sociales para los usuarios que acceden al centro comercial como lugar en el que se llevan a cabo los rituales de una sociabilidad distinguida y signada por los valores de la distinción. 
De ahí que se perciba mayor consciencia del diseño, y que a éste se le adjudique un mayor valor entre los usuarios del Centro Comercial Unicentro (asociado en nuestra triada con los estratos socieconómicos altos), quienes se apropian de este espacio modelizándolo principalmente como lugar de encuentro y esparcimiento, en el que la belleza, la comodidad, la seguridad, y la sensación de exclusividad y reconocimiento entre miembros de grupos selectos (como ocurre con la relación entre clientes y "marcas" tradicionales) que son mediados por la preponderancia del diseño, operan como las variables cualitativas que más pesan en la decisión de la visita o la compra.

En contraste, entre los usuarios del Supercentro Comercial La 14 de Calima, la visita al Mall está más inclinada a responder a necesidades de abastecimiento de productos básicos pertenecientes a la canasta familiar. En este espacio, el efecto ordenador del diseño se ve neutralizado, en buena medida, por las presiones socioeconómicas y culturales que pesan sobre los habitantes del sector en que está ubicado el centro comercial.

Lo anterior no quiere decir que el diseño no cumpla, al igual que en Unicentro, una función de mediador de las sociabilidades y de las relaciones de mercado, así como también la función de variable importante en el marco de las prácticas de consumo. Pero es claro a la luz de los datos cualitativos (reportes de los usuarios del centro comercial) y cuantitativos (agregados de ventas) que aquí se lo subordina al factor económico (tanto del lado de los vendedores como del lado de los clientes), otorgándole un papel más discreto que el que cumple en los otros dos Centros Comerciales.

Por su parte, en Cosmocentro, del cual puede decirse que representa un punto intermedio, en términos socio-económicos y espaciales, entre los dos centros comerciales anteriores, se puede apreciar cómo el diseño es mediador de la comodidad y la practicidad, pero su dimensión ligada a la exclusividad se ve menoscabada por causa de una voluntad empresarial y comercial que trata de sacar el mayor provecho económico posible del espacio disponible.

De este panorama general, descrito para cada uno de los centros comerciales, se puede colegir que en la articulación entre las prácticas comerciales-arquitectónicas y las prácticas de consumo, se encuentran unas prácticas del espacio mediadas por el Diseño y los objetos que éste produce. Estas relaciones no son unidireccionales, y es evidente que los usuarios del Mall influyen, por medio de sus prácticas (de consumo, 
de sociabilidad) y su respuesta al diseño, a su vez en la determinación del carácter del mismo y de sus espacios.

Por último, en el caso de Unicentro, en el que la función mediadora del Diseño es más evidente, se manifiesta también de forma más clara una constante propia del principio de organización de los centros comerciales en tanto que macro objetos en el que coinciden prácticas mercantiles y de sociabilidad. El local es la unidad transicional entre los niveles micro y macro-objetuales y en éste las distintas dimensiones de la práctica diseñadora se articulan para producir un efecto de Ethos en el que radica la diferencia con respecto a los competidores y el resto de los locales circundantes.

La operación de diseño tiene la función, a este nivel, de reafirmar y condensar visualmente el capital simbólico de la firma (o marca) relacionada con el espacio del local y del centro comercial, pero también con una tradición, que trasciende las barreras físicas del espacio arquitectónico-comercial concreto.

Esta operación supone la actualización consciente y estratégica de la generación de una estética que se relaciona -en el marco de referencia de una tradición- con la firma o marca, que deviene ícono y símbolo inserto en el universo isotópico de los valores ligados al de la exclusividad.

Debido a ello el espacio arquitectónico del local puede ser entendido como una retícula en la que se disponen los elementos y la sintaxis (organizada con los microobjetos y los objetos equivalentes que son contenidos por el espacio comercial) que definen un estilo previamente desarrollado y asumido por los usuarios como una unidad cultural plenamente establecida con la que se tiene una relación de reconocimiento e identificación (Berger, Luckmann, 2001).

Este estilo, en la medida de lo posible debe diferenciarse de aquél que despliegan los restantes locales, sin que entre en conflicto con el repertorio de formas del espacio arquitectónico del centro comercial. Tal diferencia suele conseguirse por medio de los materiales de la fachada, el logo de la marca, y, en términos compositivos, por la producción de un repertorio de formas propio en el que concurren los valores de lo clásico y lo moderno, fundamentales para la constitución de la identidad del Mall. 
Significados que los usuarios dan a los objetos de diseño en tres centros comerciales de Cali desde la perspectiva del consumo cultural

El objetivo de este proyecto fue la descripción de las prácticas culturales y los procesos de socialización que caracterizan la relación entre los usuarios y los objetos de diseño en los mismos tres centros comerciales estudiados en el proyecto anterior.

Para ello se hacía necesario ahondar un poco más en las semiosis que, en tanto que usuarios del espacio arquitectónico-comercial (macro-objeto de diseño), lo agentes humanos llevan a cabo con los objetos que se les ofrecen, imponen o proponen al interior del sistema del centro comercial. Por tanto, el énfasis en la indagación estuvo puesto en los significados que eran atribuidos a los objetos de diseño en las interacciones cotidianas que tienen lugar en el Mall.

Los datos para esta investigación socio-semiótica fueron obtenidos a partir de la aplicación de una encuesta y una serie de entrevistas realizadas a los usuarios de los centros comerciales. Por medio de estos instrumentos y de la observación etnográfica se pudo establecer un esquema general de dinámicas de apropiación, actualización, significación y socialización de sentidos que tiene lugar en el espacio del centro comercial, y que varía según los públicos y la modalidad de su interacción con el diseño (la cual fue abordada en la primera investigación) (Duque, 2001).

Se encontró que la experiencia de los visitantes de los centros comerciales reviste básicamente la forma de un conjunto de operaciones de actualización de los significados y los sentidos referidos o susceptibles de ser interpretados a través de los objetos de diseño. Estos actúan, en primera instancia, como señales (Eco, 2000) cuya función semiótica está regulada por leyes combinatorias internas, propias del código semiótico que se ha constituido al interior del Mall en el transcurso de los años, y que determina las posibilidades de la conducta, así como también las diversas modalidades de uso que se hacen del espacio y los elementos que contiene.

Así mismo, los objetos de diseño son operados por sus usuarios como índices que "indican" los itinerarios posibles del centro comercial, y con estos, los modos de disposición de las actividades, los consumos y los encuentros. En este sentido, los objetos de diseño, incluyendo los que son "propios" de los usuarios, sirven para "denotar" y "puntuar" órdenes de sociabilidad que han sido desarrollados específicamente para estos 
espacios, aunque son producto de la evolución de prácticas comerciales y de intercambio más tradicionales que, otrora, solían tener lugar en otros sectores de la ciudad, tales como el centro, la plaza pública, o el café.

Sin embargo, en el contexto del centro comercial, las señales que emiten los objetos de diseño en su función de marcadores del espacio y la conducta, son normativas sólo hasta cierto punto; esto se debe principalmente a que el sistema que las estructura no es de tipo cerrado, es decir, no está constituido sobre la base de una identificación exacta y unívoca entre significantes y sus significados convencionales.

Lo que en rigor sucede es que los objetos, los elementos del espacio arquitectónico, los mensajes verbales (orales y escritos) e icónicos, y las soluciones de diseño que se ofrecen a los usuarios y visitantes del centro comercial sirven a la configuración constante de variables sintácticas diversas, dependientes de la intencionalidad de esos mismos usuarios, de sus saberes previos acerca del diseño, y de su grado de inserción en las sociabilidades mediadas por los objetos de diseño orientados a generar la experiencia del confort y la exclusividad.

Los usuarios, en el entramado de este sistema de producción de sentidos que cuenta con los objetos de diseño como sus elementos mínimos visibles, reorganizan lo que se les ofrece, orientando el resultado a la satisfacción de sus propias necesidades. El influjo de los códigos y subcódigos que conforman la experiencia ideal del centro comercial (la que subyace a la intención que lo ha diseñado estratégicamente); de los constreñimientos sociales y económicos, y de las diversas variables inesperadas que introducen un principio de desviación con respecto a lo estipulado por el plano del centro comercial, son elementos que confluyen para configurar los sentidos del uso y la apropiación, que son, siguiendo a Verón, los que en definitiva actualizan lo social del diseño en el centro comercial, y la inscripción de ambos, centro comercial y diseño, en lo social.

De esta manera al sistema sintáctico que se elabora con la configuración de los objetos de diseño en y por el Mall, le corresponde un sistema semántico que se estructura en dos niveles. El primero es el que compete al marco de referencia en el que se encuentran los diferentes usos de centro comercial y objetos de diseño contenidos en este. Este sistema es el soporte y la manifestación de una gramática del espacio y la conducta que ha sido prevista y provista por el trabajo conjunto (y no siempre armónico) de los diseñadores, arquitectos y administradores de los centros comerciales. 
Dicha gramática se media y concreta en enunciados acerca de los usos del Mall que están constituidos por la combinatoria que describe la disposición espacial de los objetos de diseño y por las relaciones que estos establecen entre sí y con el espacio. Tales enunciados se manifiestan para el usuario como sobreentendidos, es decir, como un código del uso y el comportamiento que, como se vio en el proyecto anterior se actualiza de diferente forma en cada uno de los tres centros comerciales estudiados.

Estas actualizaciones diversas de la noción del Mall o centro comercial son el resultado de las operaciones que competen al segundo sistema que está en juego, de carácter semántico, el cual se compone de las múltiples redes de atribuciones de significado, apropiaciones y renuncias realizadas por los usuarios, quienes de esta manera, y por medio de sus acciones con, por y a través de los objetos, se insertan en flujos que se entrecruzan o divergen, y que están estructurados por un sinnúmero de prácticas, las cuales son los elementos fundamentales de las múltiples formas de sociabilidad que tienen lugar en los espacios estudiados. Es en estas prácticas concretas en donde se puede, para cada caso, situar la función, significación y relevancia relativa del significante "diseño" en el contexto del centro comercial.

La significación del centro comercial es, pues, siempre el producto variable pero afincado en un código normativo (en buena medida producto del diseño), de la tensión y negociación constantes entre las búsquedas y apropiaciones de los usuarios particulares y las determinaciones del pensamiento estratégico al cual el espacio debe su carácter comercial, privado y de sociabilidad ligada al consumo y la mayor o menor exclusividad de su oferta mercantil.

En consecuencia, la respuesta que el código que da su coherencia a cada centro comercial no se dan por sentadas, sino que son posibilidades en torno a las cuales orbitan las acciones concretas de los agentes humanos. La regla o norma arquitectónica, comercial y de diseño opera efectivamente pero su actualización no es un remedo perfecto de su contenido.

De esta manera, el centro comercial, aparece como un microsistema social en el que la confluencia en el espacio privado modelizado como público de personas privadas y firmas comerciales da cuenta de la dinámica esencial del poder en nuestras sociedades: es articulación y constreñimiento que permite el encuentro (que no es necesariamente sociabilidad íntima) de múltiples prácticas cuya razón de ser está en la desviación y la diferencia anóni- 
mas, a través de las cuales se manifiestan los individuos en confluencia por la satisfacción de necesidades personales o de grupos restringidos y muy localizados.

En este sentido, la consideración conjunta de lo que acontece en los tres centros comerciales permite identificar la manera en que un mismo código (sistema de significaciones) arquitectónico, comercial y de diseño, es modificado en cada caso por la inserción particular de cada uno en la cuadrícula de la ciudad; $y$, en segunda instancia, por los haceres particulares de los usuarios, que describen itinerarios orientados hacia la actualización de diversos sistemas de valores sobre la base de los cuales se constituyen la experiencia subjetiva y las modalidades de sociabilidad e intercambio (Rosanvallon, 1995). Sin embargo, es en esta profusión de minúsculas desviaciones que estriba la vida misma del Mall y su permanencia en el tiempo. El Diseño, es el mediador que posibilita la emergencia de los sentidos y la coherencia social del marco de referencia en el que dicha emergencia se presenta a diario.

En otras palabras, el diseño y sus objetos configuran una matriz estructural que es garante y condición de posibilidad de la emergencia y manifestación (actualización) de las diferencias y los reconocimientos en torno a los cuáles pivotan las demás prácticas sociales en el espacio de los centros comerciales. Esta diferencia y estos reconocimientos llegan a estar tan interiorizados que en muchos casos no son objeto de verbalización y se expresan mediante actividades ligadas a los sistemas de valores que se encarnan en cada centro comercial y en cada local.

De ahí que las elecciones y selecciones de objetos de diseño y usos de los mismos que llevan a cabo los usuarios en este marco de referencia estén orientadas principalmente ya sea por un sentido práctico (Supercentro La 14 de Calima) o por una marcada consciencia del diseño y un saber-hacer con la inversión del tiempo de ocio en función del goce que pueden procurar sociabilidades constituidas, en buena medida, en torno a la cultura letrada y a un saber informado a propósito de la dimensión estética de la experiencia (Unicentro).

\section{Usos y significados de la plaza en el espacio público y en el centro comercial.}

Análisis de un desplazamiento en una perspectiva social y política

Esta tercera investigación, de carácter cualitativo y descriptivo, y con un marcado corte etnográfico, se nutre de los hallazgos de las anteriores, y está orientada a la presentación de las diferencias que, en términos de sociabilidad se establecen en el espacio de la Plaza 
entre sus encarnaciones en el espacio público de la ciudad y el privado del comercio organizado (Yori, 2007), aún se encuentra en curso.

En este caso, la observación etnográfica es la técnica fundamental de la recolección de los datos, dado que el objetivo es elucidar la forma en que la plaza (en ambas modalidades), en tanto que objeto de diseño destinado al encuentro de los ciudadanos o de los clientes (modelización propia del agente humano en el centro comercial) -y con ella los objetos de diseño que contiene- es apropiada, usada y semantizada a través de esos usos por sus usuarios (Viviescas, 2000).

Una segunda inquietud, eminentemente sociológica nos lleva a internarnos en este campo del análisis. Ésta es la que compete a los desplazamientos de sentido operados sobre el significante /Plaza/ que pueden ser observados por medio de la comparación de lo observado en ambos espacios.

Estos desplazamientos son el síntoma de un cambio social que se está realizando ante nuestros ojos, al tiempo que formas nuevas de sociabilidad (visibles en el centro comercial) y formas tradicionales (las de la plaza pública) se traslapan y coexisten, aunque separadas espacialmente en distintos puntos de la ciudad y referidos a poblaciones diversas (Silva, 2002).

Los lugares concretos de observación para este estudio son el Centro Comercial Unicentro y la Plaza Pública de Caycedo. Esta elección se ha basado en los resultados de los anteriores proyectos, que nos permitieron identificar al mencionado centro comercial como aquel en el que se marca una más acendrada mediación del diseño y de las prácticas contemporáneas del Mall.

Los avances metodológicos que se han llevado a cabo, consistentes en la realización de extensas jornadas de observación en uno y otro espacio, y en diversas horas y días de la semana, se concretan en sendos relatos de las dinámicas de interacción entre los agentes humanos y con su espacio, a través de los cuales es posible adelantar algunos elementos fundamentales para la descripción, entre los que, acaso el más relevante, sea el que compete a la identificación de dos modalidades de uso diversas que están en estrecha relación con el carácter de cada una de las plazas.

En la Plaza Pública, otrora centro del comercio, del poder político y eclesiástico, de la reunión pública cotidiana, el rumor y la difusión de las noticias y acontecimientos de 
importancia social y personal, los usuarios del espacio se dividen, por lo general, en cuatro categorías: comerciantes informales, servidores públicos (policía y guardas cívicos), viandantes y asiduos (Pérgolis, 2000).

Excepto por los viandantes, que usan la plaza como lugar de paso, las otras tres categorías describen itinerarios de acción y reconocimiento que dan cuenta de una familiaridad producto del encuentro cotidiano, y de una forma de habitar el espacio público (que es de todos, y de nadie) durante las horas diurnas extraña al centro comercial.

El tiempo que se pasa en la Plaza pública se dedica a la conversación entre individuos que no están constreñidos por las presiones del horario diurno de trabajo o de estudio (desempleados, jubilados, trabajadores a destajo, etc.), a diferencia de lo que ocurre en el centro comercial, en el que, los flujos se diferencian claramente: en las horas de oficina o de estudio la concurrencia es poca, y tiende a aumentar al término de la jornada.

Así mismo, en la plaza pública el diseño arquitectónico (disposición de bancas, rebordes, prados, árboles, pasillos) es apropiado de manera espontánea para la realización de las actividades comerciales informales y del tiempo de ocio que allí tienen lugar. Complementariamente, los objetos de que hacen uso los presentes (especialmente las vendedoras de café, dulces y comidas) no llevan inscrita la marca del diseño profesional acordado a las funciones para las que han sido reciclados o transformados. Es posible ver como un coche de bebé se transforma, en función de la necesidad, en un carro de venta, o como los rebordes de las fuentes son apropiados como espacio para sentarse y dar inicio a animadas charlas que se extienden durante horas.

No hay prisa en la Plaza Pública. Sus asiduos-habitantes no tienen a donde más ir, y aquí se encuentran para pasar las horas en compañía de sus amigos. El ritual cotidiano de su encuentro es básicamente similar en todos los rincones de la Plaza: el que llega es llamado efusivamente por los demás. Las manos tocan los cuerpos para acentuar una frase ingeniosa, o los efectos humorísticos de una anécdota relativa a las peripecias de la vida cotidiana. En estas dinámicas participan por igual policías, vagabundos, vendedores ambulantes y participantes en cada uno de los corrillos que se arman a lo largo de la Plaza.

La risa, muchas veces estentórea, pagada de sí misma, desinhibida, es el marcador privilegiado que inicia o termina los períodos del intercambio verbal. Las apelaciones entre los presentes, los llamados, se modelizan en el orden de la picaresca, la burla, la 
familiaridad chabacana propia de las clases más populares, en las que los códigos de comportamiento no están tan permeados por el mandato del autocontrol, la distancia de los cuerpos, y los valores burgueses de la cortesía, como en las clases más altas, a las cuales se las ve pasar por las sillas de los cafés de la Nueva Plaza de Unicentro solamente durante un rato mientras se realiza una compra o una diligencia bancaria, sin perder el control y el decoro que el lugar y el hábito imponen.

En la plaza pública falta todo, y no falta nada. A nadie parecen importarle los monumentos ni la ausencia de equipamiento cómodo o de espacios que sugieran privacidad y exclusividad. Todos se las arreglan con lo que tienen a mano, mientras tras la barrera física y simbólica de los bordes de la plaza, otra ciudad (que no obstante es la misma) se afana en sus deberes y hace uso de los objetos de diseño de cafés, oficinas bancarias y de negocios, con una prisa ajena a la tranquilidad parsimoniosa de aquellos que se encuentran día tras día a ganarse la vida con el comercio informal, a vigilar el espacio en el que ya son reconocidos como parte del grupo, o simplemente a ver pasar las horas mientras ejercen prácticas de sociabilidad que parecerían al ojo desprovisto de una perspectiva histórica y sociológica del cambio, propias de tiempos hace mucho idos (Camps, 2001).

En la Plazoleta del Oasis en Unicentro, por el contrario, los usuarios no son habitantes, sino visitantes. Su estancia está mediada y determinada por las dinámicas del trabajo o el estudio; por la gramática de la conducta que ayuda a la actualización de un habitus de clase basado en el autocontrol y la mesura; por la gramática del espacio que ofrece la comodidad, la exclusividad y la practicidad que denotan los objetos de diseño que articulan el espacio (incluyendo a la propia fuente).

En consecuencia, un sistema de valores opuesto al de la plaza pública es el que estructura las prácticas, los usos del espacio y los objetos de diseño en el centro comercial. Aquí el sujeto está más distante de los otros, y el espacio adquiere un cariz más instrumental: es el lugar de encuentro o de la espera en medio de otras actividades en el que no se busca otra cosa, y en el que no se consume demasiado tiempo de una vida ya de por sí atareada.

En dicho espacio, la arquitectura, los locales asumidos como objetos de diseño y sus marcas distintivas, los micro-objetos que componen el arsenal de la atención al público y el equipamiento de los diversos sitios que ofrecen sus servicios a los clientes, cumplen 
la función de signar la experiencia e inscribirla en el sistema de valores acordado socialmente al consumo suntuario.

Cada objeto manipulado, cada milímetro de la plazoleta es portadora de un mensaje que vincula al usuario con el universo de los que saben discernir el valor relevante del diseño en la modelización estética de la experiencia humana. Pero este privilegio se da al costo de expulsar de las interacciones la calma, la risa constante, el desenfado y la despreocupación que reinan en la plaza pública.

\section{Conclusión}

Los resultados del trabajo hasta aquí expuesto permiten avizorar que, en el cruce entre diseño y sociología asumidos como prácticas que generan sentidos y como modos de interpretación y mediadores de la comprensión de las dinámicas sociales, se abre un campo de estudio promisorio para la disciplina.

El recurso a una perspectiva de análisis iconológico, y a otra iconográfica, de los objetos; la utilización de técnicas mixtas de investigación; y la articulación del análisis semiológico en el arsenal del estudio sociológico se han revelado como vías fructíferas para el desvelamiento y decodificación de procesos que en este mismo instante corren curso en nuestra sociedad, y cuya comprensión resulta capital para el abordaje de los retos que se le plantean a la disciplina en un mundo contemporáneo en el que las formas del cambio se han diversificado hasta tal punto, que buena parte de la práctica sociológica tradicional empieza a resultar insuficiente para dar cuenta de la materia de lo social.

El Seminario de Sociología del Diseño continuará explorando esta senda que se ha abierto gracias a la afortunada identificación temprana que en su seno se realizó de la función del diseño como mediador social; de las diversas modalidades en que éste opera como dinamizador, productor, conformador e instrumento de lo social; y de las también diversas maneras en que lo social del diseño no se restringe a discursos o prácticas localizadas sino que es un principio de ordenamiento de la sociedad al que nuestra disciplina está llamada a prestar más atención.

Por nuestra parte, aún resta mucho por hacer en lo que compete al estudio de la relación que se establece entre esos cascarones que son los macro-objetos de diseño del tipo de los centros comerciales, y los flujos que en estos tienen lugar. Nuestros próximos 
proyectos, muy seguramente, virarán en esta dirección que nos introduce en las teorías y metodologías de sistemas dinámicos, y con base en las cuales, estamos seguros, podemos avanzar un poco más en la diagramación de las complejas prácticas que estructuran los sentidos de lo social a partir del influjo del diseño en nuestras vidas.

\section{Bibliografía}

Berger, Peter y Luckmann, Thomas (2001). La construcción social de la realidad. Buenos Aires. Amorrortu editores.

Camps, Victoria (2000). Elementos históricos del concepto de lo público. Cartagena, Memorias del V encuentro iberoamericano del tercer sector.

De Certeau, Michel (2000). La invención de lo cotidiano. México: Universidad Iberoamericana.

Delgado, Manuel (1999). El animal público. Barcelona, Editorial Anagrama.

Duque, Félix (2001). Arte público y espacio político. Madrid. Editorial Akal.

Eco, Umberto (2000). Tratado de semiótica general. Barcelona, Editorial Lumen.

Guerrero Caicedo, Mauricio (ed.) (2014). Objetos públicos, espacios privados: usuarios y relaciones sociales en tres centros comerciales de Santiago de Cali. Cali: Editorial Universidad Icesi

Heller, Agnes (1994). Sociología de la Vida Cotidiana. Barcelona, Editorial Península.

Latour, Bruno (2008). Reensamblar lo social. Buenos Aires,Editorial Manantial.

Moles, Abraham (1975). Teoría de los Objetos. Barcelona, Editorial Gustavo Gili.

Pérgolis, Juan Carlos (2000). La Ciudad: hábitat de diversidad y complejidad. Bogotá, Universidad Nacional de Colombia.

Rosanvallon, Pierre (1995). La nueva cuestión social. Buenos Aires, Ediciones Manantial.

Sánchez, Mauricio (2001). Morfogénesis del Objeto de Uso. Bogotá, Universidad Jorge Tadeo Lozano.

Silva, Armando (2002). Estrategias estético-políticas en el espacio público contemporá- 
neo. Gaceta del Ministerio de Cultura, Número 44, Bogotá.

Verón, Eliseo (1993). La semiosis social. Barcelona, Editorial Gedisa.

Viviescas, Fernando (2000). El espacio público de la ciudad. Revista Universidad de Medellín número 67, Medellín.

Yorí, Carlos Mario (2007). Ciudad, ciudadanía y espacio público. Bogotá. Editorial Pontificia Universidad Javeriana 